\title{
Global Attractor of Solutions of a Rational System in the Plane
}

\author{
Miron B. Bekker, ${ }^{1}$ Martin J. Bohner, ${ }^{2}$ and Hristo D. Voulov ${ }^{3}$ \\ ${ }^{1}$ Department of Mathematics, University of Pittsburgh at Johnstown, Johnstown, PA, USA \\ ${ }^{2}$ Department of Mathematics and Statistics, Missouri University of Science and Technology, Rolla, MO, USA \\ ${ }^{3}$ Department of Mathematics and Statistics, University of Missouri-Kansas City, Kansas City, MO, USA
}

Correspondence should be addressed to Martin J. Bohner; bohner@mst.edu

Received 22 March 2015; Revised 14 July 2015; Accepted 15 July 2015

Academic Editor: Antonia Vecchio

Copyright (C) 2015 Miron B. Bekker et al. This is an open access article distributed under the Creative Commons Attribution License, which permits unrestricted use, distribution, and reproduction in any medium, provided the original work is properly cited.

We consider a two-dimensional autonomous system of rational difference equations with three positive parameters. It was conjectured that every positive solution of this system converges to a finite limit. Here we confirm this conjecture, subject to an additional assumption.

\section{Introduction}

Rational systems of first-order difference equations in the plane have been receiving increasing attention in the last decade. Recently, in [1-15] (see also the references therein), efforts have been made for a more systematic approach. In particular, the rational system

$$
\begin{aligned}
& x_{n+1}=\frac{\alpha_{1}+y_{n}}{x_{n}}, \\
& y_{n+1}=\frac{\alpha_{2}+\beta_{2} x_{n}+\gamma_{2} y_{n}}{A_{2}+B_{2} x_{n}+C_{2} y_{n}}
\end{aligned}
$$

with nonnegative coefficients and initial conditions was studied in [4]. Along with the results published in [4], several conjectures about some nontrivial cases were also posed. In the case when

$$
\alpha_{1}=\alpha_{2}=\beta_{2}=0 \text {, }
$$

we have confirmed in [2] that [4, Conjecture 2.4 on page 1223] is true. Our goal here is to confirm another conjecture, in the case when

$$
\begin{aligned}
& \alpha_{1} \geq 0, \\
& \beta_{2}=C_{2}=0 .
\end{aligned}
$$

Conjecture 1 (see [4, Conjecture 2.1 on page 1217]). Let $a \geq 0$ and $p, q>0$. Then every positive solution of the system

$$
\begin{aligned}
& x_{n+1}=\frac{a+y_{n}}{x_{n}}, \\
& y_{n+1}=\frac{p+y_{n}}{q+x_{n}}
\end{aligned}
$$

converges to a finite limit.

By utilizing the relation

$$
y_{n}=x_{n} x_{n+1}-a, \quad n \in \mathbb{N}_{0},
$$

it is clear that the $x$-component of any positive solution $\left\{\left(x_{n}, y_{n}\right)\right\}_{n \in \mathbb{N}_{0}}$ of (4) must satisfy the inequality $x_{n} x_{n+1}>a$ as well as the difference equation

$$
x_{n+2}=f\left(x_{n+1}, x_{n}\right), \quad n \in \mathbb{N}_{0},
$$

where the function $f$ is defined by

$$
f(u, v)=\frac{u v+a v+p+a q-a}{u(q+v)} .
$$

We consider the open subset $G$ of the first quadrant defined by

$$
G=\{(u, v): u, v>0, u v>a\}
$$


and the map $T$ defined on $G$ by

$$
T(u, v)=(f(u, v), u), \quad(u, v) \in G .
$$

It is easy to see that $G$ is invariant for $T$, that is, $T(G) \subset G$, and that $T$ has a unique fixed point $\left(x^{*}, x^{*}\right)$ in $G$. We will prove that, in the case

$$
\frac{p}{2} \leq a+(q+1)(1+\sqrt{a+1})
$$

every solution $\left\{x_{n}\right\}_{n \in \mathbb{N}_{0}}$ of (6), with positive initial values $x_{0}$ and $x_{1}$ such that $x_{0} x_{1}>a$, is positive, satisfies the inequality $x_{n} x_{n+1}>a$ for all $n \in \mathbb{N}_{0}$, and converges to the equilibrium $x^{*}$. Thus, the fixed point $\left(x^{*}, x^{*}\right)$ is a global attractor for all trajectories $\left\{T^{n}(u, v)\right\}_{n \in \mathbb{N}_{0}}$ with initial point $(u, v) \in G$. Then, in view of (5), the point $\left(x^{*}, x^{* 2}-a\right)$ is a global attractor for all positive solutions of (4), which confirms the conjecture in case (10) holds.

\section{Preliminaries}

We recall the following three useful theorems.

Theorem 2 (see [5, page 11]). Let $I \subset \mathbb{R}$ and suppose that $F: I \times I \rightarrow I$ is nonincreasing in the first variable and nondecreasing in the second variable. Then, for every solution $\left\{x_{n}\right\}_{n \in \mathbb{N}_{0}}$ of

$$
x_{n+2}=F\left(x_{n+1}, x_{n}\right), \quad n \in \mathbb{N}_{0} \text {, }
$$

both subsequences $\left\{x_{2 n}\right\}_{n \in \mathbb{N}_{0}}$ and $\left\{x_{2 n+1}\right\}_{n \in \mathbb{N}_{0}}$ are eventually monotone.

Theorem 3 (see [3]). Every positive solution of (4) is bounded.

Theorem 4 (see [11, Theorem 1.4 .7 on page 13]). Let $I \subset \mathbb{R}$ be a bounded interval and suppose that $f: I \times I \rightarrow I$ is continuous and nonincreasing in each of its arguments. Assume that if $m, M \in I$ with $f(m, m)=M$ and $f(M, M)=m$, then $m=M$. Then there exists $x^{*} \in I$ such that every solution of $x_{n+2}=f\left(x_{n+1}, x_{n}\right)$ converges to $x^{*}$.

For the proof of our main result in the next section, we also use the following auxiliary result.

Lemma 5. Let $a \geq 0$ and $p, q>0$. Let $f(u, v)$ be defined by (7) for $u>0, v \geq 0$, and define

$$
\begin{aligned}
& A=p-a+a q, \\
& B=p-a .
\end{aligned}
$$

Then the following statements are true:

(i) $f(u, v)$ is decreasing in $u$ provided av $+A>0$, and $f(u, v)$ is increasing in $u$ provided av $+A<0$.

(ii) $f(u, v)$ is increasing in $v$ provided $q u>B$, and $f(u, v)$ is decreasing in $v$ provided $q u<B$.

(iii) If $u v>a$, then $f(u, v) u>a$. (iv) If $\left\{x_{n}\right\}_{n \in \mathbb{N}_{0}}$ is a positive solution of (6) such that $x_{0} x_{1}>$ $a$, then $y_{n}=x_{n+1} x_{n}-a>0$ for all $n \in \mathbb{N}_{0}$, and the sequence $\left\{\left(x_{n}, y_{n}\right)\right\}_{n \in \mathbb{N}_{0}}$ is a positive and bounded solution of (4).

(v) If $B, u, v>0$ and $u q \neq B$, then

$$
\begin{array}{r}
\left(u-B q^{-1}\right)\left(f(u, v)-A B^{-1}\right)<0, \\
\left(u-B q^{-1}\right)\left(f(u, v)-A(q u)^{-1}\right)>0 .
\end{array}
$$

Proof. By direct computation of the partial derivatives

$$
\begin{aligned}
& \frac{\partial f}{\partial u}(u, v)=\frac{a-p-a q-a v}{(q+v) u^{2}} \\
& \frac{\partial f}{\partial v}(u, v)=\frac{a-p+q u}{u(q+v)^{2}}
\end{aligned}
$$

the proofs of (i) and (ii) follow. From (7), we obtain

$$
\begin{aligned}
u f(u, v)-a & =\frac{u v+a v+p+a q-a}{q+v}-a \\
& =\frac{u v-a+p}{q+v}
\end{aligned}
$$

which implies (iii). The proof of (iv) follows from (iii), (6), and Theorem 3. It remains to prove (v). Since $A=B+a q>0$, (i) implies that $f(u, v)$ is decreasing in $u$, and (13) follows from $f\left(B q^{-1}, v\right)=A B^{-1}$. Finally, for $u>B q^{-1}$, it follows from (ii) that $f(u, v)$ is increasing in $v$ and $f(u, v)>f(u, 0)=A(q u)^{-1}$. Similarly, for $u<B q^{-1}$, it follows from (ii) that $f(u, v)$ is decreasing in $v$ and $f(u, v)<f(u, 0)=A(q u)^{-1}$. Thus, (14) follows and the proof is complete.

\section{Main Results}

Lemma 6. Let $a \geq 0$ and $p, q>0$. Let $f$ be defined by (7) and let $\left\{x_{n}\right\}_{n \in \mathbb{N}_{0}}$ be a positive solution of (6) such that $x_{0} x_{1}>a$. Assume that the subsequence $\left\{x_{2 n}\right\}_{n \in \mathbb{N}_{0}}$ converges to $x_{\mathrm{e}}>0$. Then the following statements are true:

(i) The subsequence $\left\{x_{2 n+1}\right\}_{n \in \mathbb{N}_{0}}$ converges to some $x_{0}>$ $a x_{\mathrm{e}}^{-1}$.

(ii) The sequence $\left\{x_{n}\right\}_{n \in \mathbb{N}_{0}}$ converges to some $x^{*}$, and $x^{*}$ is the unique root of the characteristic equation

$$
\lambda^{3}+\lambda^{2}(q-1)-a \lambda+a-a q-p=0
$$

such that $x^{*}>\max \{\sqrt{a}, 1-q\}$.

Proof. We first show (i). It follows from Lemma 5(iv) that the sequence $\left\{x_{n}\right\}_{n \in \mathbb{N}_{0}}$ is bounded. In view of (6) and (7), we have

$$
x_{2 n+1}\left[x_{2 n+2}\left(q+x_{2 n}\right)-x_{2 n}\right]=a x_{2 n}-a+a q+p .
$$

If we suppose that $x_{\mathrm{e}}=1-q$, then from (18) and the boundedness of $\left\{x_{2 n+1}\right\}_{n \in \mathbb{N}_{0}}$, by letting $n \rightarrow \infty$, we obtain 
$0=a x_{\mathrm{e}}-a(1-q)+p=p$, which is a contradiction. Hence, $x_{\mathrm{e}} \neq 1-q$, and, from (18), it follows that

$$
\begin{aligned}
0 & \leq \lim _{n \rightarrow \infty} x_{2 n+1}=\frac{a x_{\mathrm{e}}-a+a q+p}{x_{\mathrm{e}}\left(q+x_{\mathrm{e}}-1\right)} \\
& =: x_{\mathrm{o}}, \\
x_{\mathrm{o}} x_{\mathrm{e}}\left(q+x_{\mathrm{e}}-1\right) & =a\left(x_{\mathrm{e}}+q-1\right)+p .
\end{aligned}
$$

Since $x_{0} x_{1}>a$, by Lemma 5(iii), it follows that $x_{2 n+1} x_{2 n}>a$ for all $n \in \mathbb{N}_{0}$ and, by letting $n \rightarrow \infty$, we obtain $x_{\mathrm{o}} x_{\mathrm{e}} \geq a$. If we suppose that $x_{\mathrm{o}} x_{\mathrm{e}}=a$, then (20) implies $p=0$, which is a contradiction. Therefore, $x_{\mathrm{o}} x_{\mathrm{e}}>a \geq 0$. The proof of (i) is complete.

Now we show (ii). Since the sequence $\left\{x_{n+1}\right\}_{n \in \mathbb{N}_{0}}$ is also a positive solution of (6) such that $x_{0} x_{1}>a$, it follows from (i) that besides (20) we also have

$$
x_{\mathrm{e}} x_{\mathrm{o}}\left(q+x_{\mathrm{o}}-1\right)=a\left(x_{\mathrm{o}}+q-1\right)+p .
$$

By subtracting (21) from (20), we obtain

$$
x_{\mathrm{e}} x_{\mathrm{o}}\left(x_{\mathrm{e}}-x_{\mathrm{o}}\right)=a\left(x_{\mathrm{e}}-x_{\mathrm{o}}\right)
$$

and, in view of $x_{\mathrm{e}} x_{\mathrm{o}}>a$, this yields $x_{\mathrm{e}}=x_{\mathrm{o}}=: x^{*}$. Therefore,

$$
\lim _{n \rightarrow \infty} x_{n}=x^{*}>\sqrt{a}
$$

and (21) implies that $x^{*}$ is a zero of the characteristic polynomial

$$
g(\lambda):=\lambda^{3}+(q-1) \lambda^{2}-a \lambda+a(1-q)-p .
$$

Observe that $g$ has a unique root $x^{*}$ in the interval $(\sqrt{a}, \infty)$, and the inequality $x^{*}>\max \{\sqrt{a}, 1-q\}$ follows from $g(\sqrt{a})=$ $g(1-q)=-p<0$. The proof is complete.

Lemma 7. Let $a \geq 0$ and $p, q>0$. Let $f$ be defined by (7) and let $\left\{x_{n}\right\}_{n \in \mathbb{N}_{0}}$ be a positive solution of (6) such that $x_{0} x_{1}>a$. Then there exists $n_{0} \in \mathbb{N}_{0}$ such that

$$
\begin{aligned}
& a x_{n} \geq a-a q-p, \quad n>n_{0}, \\
& x_{n}>1-q, \quad n>n_{0} .
\end{aligned}
$$

Proof. Define $A$ and $B$ by (12). For $A \geq 0$, inequality (25) is trivial. Now assume that $A<0$ that is, $0<p<a(1-q)<a$. Since $B=p-a<0$, it follows from Lemma 5(ii) that $f(u, v)$ is increasing in $v$, and $x_{n} \geq-A a^{-1}$ implies

$$
\begin{aligned}
x_{n+2} & =f\left(x_{n+1}, x_{n}\right) \geq f\left(x_{n+1},-A a^{-1}\right)=\frac{-A}{a-p} \\
& >\frac{-A}{a} .
\end{aligned}
$$

Hence, (25) will follow if each of the subsequences $\left\{x_{2 n}\right\}_{n \in \mathbb{N}_{0}}$ and $\left\{x_{2 n+1}\right\}_{n \in \mathbb{N}_{0}}$ enters the interval $\left[-A a^{-1}, \infty\right)$. For the sake of contradiction, suppose that, for some $m \in\{0,1\}$, we have

$$
x_{m+2 n} \in\left(0,-A a^{-1}\right), \quad n \in \mathbb{N}_{0} \text {. }
$$

Then, by Lemma 5(i), $f\left(u, x_{m+2 n}\right)$ is increasing in $u$, and, for all $n \in \mathbb{N}_{0}$, the inequality $x_{m+2 n+1} x_{m+2 n}>a$ implies that

$$
x_{m+2 n+2}=f\left(x_{m+2 n+1}, x_{m+2 n}\right)>f\left(a x_{m+2 n}^{-1}, x_{m+2 n}\right) \text {. }
$$

Since $p>0$, it follows that

$$
f\left(a x^{-1}, x\right)=\frac{x(a q+a x+p)}{a(q+x)}>x, \quad x>0 .
$$

In view of (29) and (30), (28) implies that the subsequence $\left\{x_{m+2 n}\right\}_{n \in \mathbb{N}_{0}}$ is increasing and must converge to some $x^{*} \in$ $\left(0,-A a^{-1}\right]$ that is,

$$
x^{*} \leq 1-q-p a^{-1}<1-q .
$$

On the other hand, Lemma 6 implies that $x^{*}>1-q$, which is a contradiction. Hence, (28) cannot be true, and the proof of (25) is complete. Then, for every $n>n_{0}$, we have

$$
x_{n+2}=f\left(x_{n+1}, x_{n}\right)=\frac{x_{n+1} x_{n}+a x_{n}+A}{x_{n+1}\left(x_{n}+q\right)} \geq \frac{x_{n}}{q+x_{n}} .
$$

If $x_{n}>1-q$ for some $n>n_{0}$, then it follows from (32) that

$$
x_{n+2} \geq \frac{x_{n}}{q+x_{n}}=1-\frac{q}{q+x_{n}}>1-q .
$$

It remains to show that both subsequences $\left\{x_{2 n}\right\}_{n \in \mathbb{N}_{0}}$ and $\left\{x_{2 n+1}\right\}_{n \in \mathbb{N}_{0}}$ eventually enter the interval $(1-q, \infty)$. For the sake of contradiction, suppose that, for some $m \in\{0,1\}$, we have

$$
x_{m+2 n} \leq 1-q, \quad n \in \mathbb{N}_{0} .
$$

Then, for every $n \in \mathbb{N}_{0}$ such that $m+2 n>n_{0}$, it follows from (32) that $x_{m+2 n+2} \geq x_{m+2 n}$. Therefore, the subsequence $\left\{x_{m+2 n}\right\}_{n \in \mathbb{N}_{0}}$ is nondecreasing and must converge to some $x^{*} \in$ $(0,1-q]$. However, Lemma 6 implies that $x^{*}>1-q$, which is a contradiction. Hence, (34) cannot be true and the proof of (26) follows. The proof is complete.

Now we are ready to prove our main result.

Theorem 8. Let $a \geq 0$ and $p, q>0$ and assume that (10) holds. Assume that $\left\{x_{n}\right\}_{n \in \mathbb{N}_{0}}$ is a positive solution of (6) such that $x_{0} x_{1}>a$. Then, $\left\{x_{n}\right\}_{n \in \mathbb{N}_{0}}$ converges to a finite limit $x^{*}$, which is the unique root of the characteristic equation (17) such that $x^{*}>\max \{\sqrt{a}, 1-q\}$.

Proof. From Lemma 5(iv), it follows that there exists $L>0$ such that $x_{n} \in(0, L)$ for all $n \in \mathbb{N}_{0}$. Then, (6) implies that

$$
x_{n+2}>\frac{p}{x_{n+1}\left(q+x_{n}\right)}>\frac{p}{L(q+L)}=b>0 \text {. }
$$

Thus,

$$
0<b<x_{n}<L, \quad n \geq 3 .
$$

First, assume that $a \geq p$. If $a-a q-p<0$, then we have that $f(u, v)>0$ for all $u, v>0$ and, by Lemma $5, f(u, v)$ is 
decreasing in $u$ and increasing in $v$. Therefore, in this case, the proof follows from (36), Theorem 2 with $I=(0, \infty)$, and Lemma 6. Now assume that $a-a q-p \geq 0$ that is, $0<p \leq$ $a(1-q)<a$. Then $q<1$, and, by Lemma 7 , we have $x_{n}>$ $1-q$ eventually. It is easy to see that $u, v \in(1-q, \infty)$ implies $f(u, v) \in(1-q, \infty)$. From the inequalities $a v>a(1-q)-p$ and $q u>q(1-q)>0 \geq p-a$, we obtain by Lemma 5 that $f(u, v)$ is decreasing in $u$ and increasing in $v$ on $(1-q, \infty)$. Hence, in this case, the proof follows from (36), Theorem 2 with $I=(1-q, \infty)$, and Lemma 6 .

Now we assume that $a<p$. Define $A$ and $B$ as in (12). Since $A \geq B>0$, from Lemma 5(v), it follows that, for all $u, v>0$,

$$
\begin{aligned}
\left(u-B q^{-1}\right)\left(f(u, v)-A B^{-1}\right) & <0 \\
& \text { provided } u \neq B q^{-1}, \\
\left(u-B q^{-1}\right)\left(f(u, v)-A(q u)^{-1}\right) & >0 \\
& \text { provided } u \neq B q^{-1} \\
f\left(B q^{-1}, v\right) & =A B^{-1} .
\end{aligned}
$$

If $B q^{-1}<u \leq A B^{-1}$ and $v>0$, then (37) and (38) imply

$$
A B^{-1}>f(u, v)>A(q u)^{-1} \geq B q^{-1}
$$

and, in view of (39), we obtain

$$
f(u, v) \in\left[B q^{-1}, A B^{-1}\right], \quad u \in\left[B q^{-1}, A B^{-1}\right], v>0 .
$$

In the same fashion, we obtain

$$
f(u, v) \in\left[A B^{-1}, B q^{-1}\right], \quad u \in\left[A B^{-1}, B q^{-1}\right], v>0 .
$$

Now, assume that $x_{m} \in\left[B q^{-1}, A B^{-1}\right]$ for some $m>1$. Then, by (41), we have

$$
x_{n} \in\left[B q^{-1}, A B^{-1}\right], \quad n \geq m
$$

From (41), Lemma 5(i)(ii), and Theorem 2 with $I=$ $\left[B q^{-1}, A B^{-1}\right]$, it follows that the subsequence $\left\{x_{2 n}\right\}_{n \in \mathbb{N}_{0}}$ is monotone. But it is also bounded, in view of (41), and must converge to some $x_{\mathrm{e}} \in[b, L]$. Therefore, by Lemma 6, we obtain $x_{\mathrm{e}}=x^{*}$ and

$$
\lim _{n \rightarrow \infty} x_{n}=x^{*}
$$

Next, assume that $x_{m} \in\left[A B^{-1}, B q^{-1}\right]$ for some $m>1$. Then, by (42), we have

$$
x_{n} \in\left[A B^{-1}, B q^{-1}\right], \quad n \geq m
$$

In this case, $I=\left[A B^{-1}, B q^{-1}\right] \neq \emptyset$, the function $f: I \times I \rightarrow$ $I$ defined by (7) is continuous, and, by Lemma 5(i)(ii), it is nonincreasing in each of its arguments. In order to verify the second condition of Theorem 4, we suppose for the sake of contradiction that there exist $m, M \in I$ with $f(m, m)=M$, $f(M, M)=m$, and $m \neq M$, which implies

$$
\begin{aligned}
(M-m)(M+m+a-m M) & =0, \\
m M & =M+m+a .
\end{aligned}
$$

Then, in view of $f(m, m)=M$, we obtain

$$
\begin{aligned}
m+M & =\frac{B-a}{q+1}, \\
m M & =\frac{A}{q+1} .
\end{aligned}
$$

Thus, $m$ and $M$ are different positive roots of the quadratic polynomial

$$
z^{2}-\frac{B-a}{q+1} z+\frac{A}{q+1} .
$$

Therefore, $B-a>0$ and $(B-a)^{2}-4 A(q+1)>0$, which contradicts (10). Since $x^{*}$ is the unique positive root of the characteristic equation (17), it follows from (42) and Theorem 4 with $I=\left[A B^{-1}, B q^{-1}\right]$ that (44) holds. It remains to prove (44) in the last case, when

$$
x_{n} \notin[c, d], \quad n \geq 2,
$$

where

$$
\begin{aligned}
& c=\min \left\{B q^{-1}, A B^{-1}\right\}, \\
& d=\max \left\{B q^{-1}, A B^{-1}\right\} .
\end{aligned}
$$

In view of Lemma 7, (36), and (49), there exists some $n_{0}$ such that

$$
x_{n} \in(m, c) \cup(d, L), \quad n \geq n_{0},
$$

where

$$
m=\max \{b, 1-q\} .
$$

Since the sequence $\left\{x_{n_{0}+n}\right\}_{n \in \mathbb{N}_{0}}$ is also a positive solution of (6), in view of (37) and (38), we may assume without loss of generality that

$$
0<m<x_{2 n}<c \leq d<x_{2 n+1}<L, \quad n \in \mathbb{N}_{0}
$$

Then, by Lemma 5(i)(ii), $f\left(x_{2 k}, v\right)$ is decreasing in $v$, while $f\left(x_{2 k+1}, v\right)$ is increasing in $v$ and $f(u, v)$ is decreasing in $u$. Hence, for every $n \in \mathbb{N}_{0}$, we obtain that

$$
\begin{aligned}
c & >x_{2 n+2}=f\left(x_{2 n+1}, x_{2 n}\right)>f\left(x_{2 n+1}, m\right) \\
& >f(L, m), \\
d & <x_{2 n+3}=f\left(x_{2 n+2}, x_{2 n+1}\right)<f\left(x_{2 n+2}, d\right) \\
& <f(m, d) .
\end{aligned}
$$


The equation $f(u, v)=z$ has a unique solution for $u$ :

$$
u=g(v, z)=\frac{a v+a q+p-a}{z(q+v)-v} \quad \text { provided } z \neq \frac{v}{q+v} .
$$

Observe that the inequality $f(u, v)<z$ is equivalent to $u>$ $g(v, z)$, provided $z(q+v)-v>0$. Since (52) and (53) imply that

$$
\begin{aligned}
& \frac{c}{m}>1 \geq \frac{1}{m+q}, \\
& d+q>1,
\end{aligned}
$$

it follows from (54) that

$$
\begin{aligned}
& x_{2 n+1}>g(m, c), \\
& x_{2 n+2}<g(d, d)
\end{aligned}
$$

$n \in \mathbb{N}_{0}$.

Set $m_{1}=m, c_{1}=c, d_{1}=d, L_{1}=L$, and, for $s \in \mathbb{N}$,

$$
\begin{aligned}
m_{s+1} & =\max \left\{m_{s}, f\left(L_{s}, m_{s}\right)\right\}, \\
c_{s+1} & =\min \left\{c_{s}, g\left(d_{s}, d_{s}\right)\right\}, \\
d_{s+1} & =\max \left\{d_{s}, g\left(m_{s}, c_{s}\right)\right\}, \\
L_{s+1} & =\min \left\{L_{s}, f\left(m_{s}, d_{s}\right)\right\} .
\end{aligned}
$$

Then, by induction, we obtain that

$$
0<m_{s}<x_{2 k}<c_{s} \leq d_{s}<x_{2 k+1}<L_{s}, \quad k \geq s .
$$

Since the sequences $\left\{m_{s}\right\},\left\{c_{s}\right\},\left\{d_{s}\right\}$, and $\left\{L_{s}\right\}$ are monotone and bounded, there exist $m^{*}, c^{*}, d^{*}, L^{*}>0$ such that

$$
\begin{aligned}
& \lim _{s \rightarrow \infty} m_{s}=m^{*}, \\
& \lim _{s \rightarrow \infty} c_{s}=c^{*}, \\
& \lim _{s \rightarrow \infty} d_{s}=d^{*}, \\
& \lim _{s \rightarrow \infty} L_{s}=L^{*} .
\end{aligned}
$$

By letting $s \rightarrow \infty$ in (58) and (59), we obtain

$$
\begin{aligned}
m^{*} & =\max \left\{m^{*}, f\left(L^{*}, m^{*}\right)\right\}, \\
c^{*} & =\min \left\{c^{*}, g\left(d^{*}, d^{*}\right)\right\}, \\
d^{*} & =\max \left\{d^{*}, g\left(m^{*}, c^{*}\right)\right\}, \\
L^{*} & =\min \left\{L^{*}, f\left(m^{*}, d^{*}\right)\right\}, \\
0 & <m^{*} \leq c^{*} \leq d^{*} \leq L^{*} .
\end{aligned}
$$

Therefore,

$$
\begin{aligned}
m^{*} & \geq f\left(L^{*}, m^{*}\right), \\
d^{*} & \geq g\left(m^{*}, c^{*}\right), \\
c^{*} & \leq g\left(d^{*}, d^{*}\right), \\
L^{*} & \leq f\left(m^{*}, d^{*}\right) .
\end{aligned}
$$

But (63) implies $L^{*} \geq g\left(m^{*}, m^{*}\right)$ and $f\left(d^{*}, m^{*}\right) \leq c^{*}$ and, in view of (64), we obtain

$$
\begin{aligned}
& f\left(d^{*}, m^{*}\right) \leq g\left(d^{*}, d^{*}\right), \\
& f\left(m^{*}, d^{*}\right) \geq\left(m^{*}, m^{*}\right) .
\end{aligned}
$$

Finally, (65), (7), and (55) imply that

$$
\begin{aligned}
& (q-1)\left(m^{*} d^{*}-a\right)+p \\
& \leq p m^{*}-(p-a) d^{*}-m^{*}\left(d^{*}\right)^{2}, \\
& (q-1)\left(d^{*} m^{*}-a\right)+p \\
& \geq p d^{*}-(p-a) m^{*}-d^{*}\left(m^{*}\right)^{2} .
\end{aligned}
$$

Thus,

$$
\begin{aligned}
& p d^{*}-(p-a) m^{*}-d^{*}\left(m^{*}\right)^{2} \\
& \leq p m^{*}-(p-a) d^{*}-m^{*}\left(d^{*}\right)^{2}, \\
& \left(2 p-a+d^{*} m^{*}\right)\left(d^{*}-m^{*}\right) \leq 0 .
\end{aligned}
$$

Hence $d^{*} \leq m^{*}$ and, in view of (62), $m^{*}=c^{*}=d^{*}$. Then, from (59), it follows that the subsequence $\left\{x_{2 n}\right\}_{n \in \mathbb{N}_{0}}$ converges to $d^{*}>0$, and the proof is complete by Lemma 6 .

Example 9. Let $a=3, q=2$, and $p=3$; that is, we consider the example of system (4):

$$
\begin{aligned}
& x_{n+1}=\frac{3+y_{n}}{x_{n}}, \\
& y_{n+1}=\frac{3+y_{n}}{2+x_{n}} .
\end{aligned}
$$

Then (10) is satisfied. The root $x^{*}$ of the characteristic equation (17), that is,

$$
\lambda^{3}+\lambda^{2}-3 \lambda-6=0
$$

satisfying $x^{*}>\max \{\sqrt{a}, 1-q\}$ is $x^{*}=2$. By Theorem 8 , if $x_{0} x_{1}>3$, then any positive solution of (68) satisfies

$$
\begin{aligned}
& \lim _{n \rightarrow \infty} x_{n}=2, \\
& \lim _{n \rightarrow \infty} y_{n}=1 .
\end{aligned}
$$

(a) Let $x_{0}=2$ and $y_{0}=1$. Then $x_{1}=2$ and $y_{1}=1$. Hence $x_{0} x_{1}=4>3$. In fact, $x_{n}=2$ and $y_{n}=1$ for all $n \in \mathbb{N}_{0}$ that is, (70) holds true.

(b) Let $x_{0}=1$ and $y_{0}=4$. Then $x_{1}=7$. Hence $x_{0} x_{1}=$ $7>3$. From Figure 1, it can be seen that (70) holds true.

(c) Let $x_{0}=4$ and $y_{0}=1$. Then $x_{1}=1$. Hence $x_{0} x_{1}=$ $4>3$. From Figure 2, it can be seen that (70) holds true. 


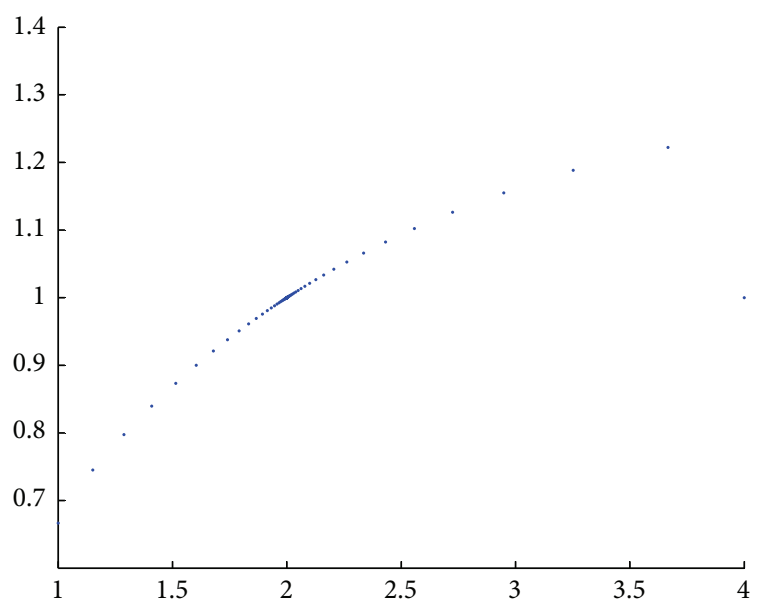

Figure 1: Plot of $\left(x_{n}, y_{n}\right)$ for Example 9(b).

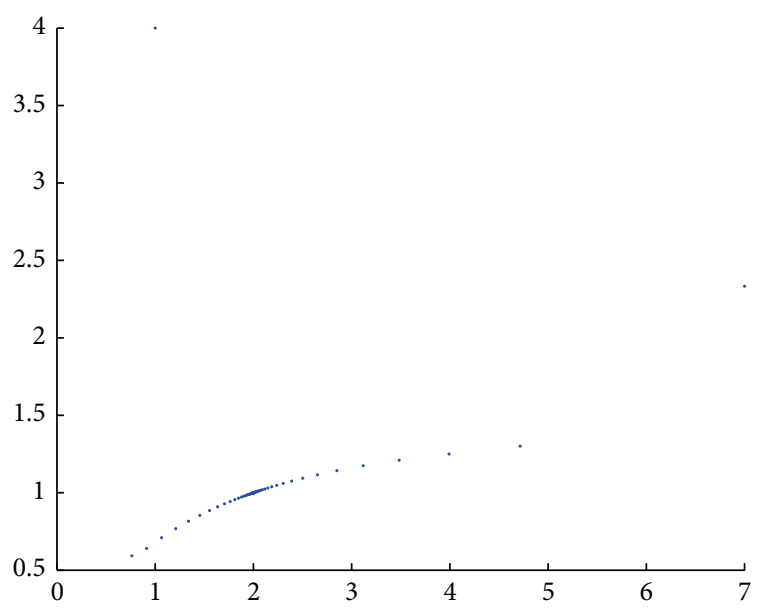

Figure 2: Plot of $\left(x_{n}, y_{n}\right)$ for Example 9(c).

\section{Conflict of Interests}

The authors declare that there is no conflict of interests regarding the publication of this paper.

\section{References}

[1] S. Atawna, R. Abu-Saris, and I. Hashim, "Local stability of period two cycles of second order rational difference equation," Discrete Dynamics in Nature and Society, vol. 2012, Article ID 969813, 11 pages, 2012.

[2] M. B. Bekker, M. J. Bohner, and H. D. Voulov, "Asymptotic behavior of solutions of a rational system of difference equations," Journal of Nonlinear Science and Its Applications, vol. 7, no. 6, pp. 379-382, 2014.

[3] E. Camouzis, E. Drymonis, and G. Ladas, "Patterns of boundedness of the rational system $x_{n+1}=\alpha_{1}+\beta_{1} x_{n} / A_{1}+C_{1} y_{n}$ and $y_{n+1}=\alpha_{2}+\beta_{2} x_{n}+\gamma_{2} y_{n} / A_{2}+B_{2} x_{n}+C_{2} y_{n}$," Fasciculi Mathematici , no. 44, pp. 9-18, 2010.

[4] E. Camouzis, C. M. Kent, G. Ladas, and C. D. Lynd, "On the global character of solutions of the system: $x_{n+1}=\alpha_{1}+y_{n} / x_{n}$ and $y_{n+1}=\alpha_{2}+\beta_{2} x_{n}+\gamma_{2} y_{n} / A_{2}+B_{2} x_{n}+C_{2} y_{n}$, Journal of Difference Equations and Applications, vol. 18, no. 7, pp. 1205-1252, 2012.

[5] E. Camouzis and G. Ladas, Dynamics of Third-Order Rational Difference Equations with Open Problems and Conjectures, vol. 5 of Advances in Discrete Mathematics and Applications, Chapman \& Hall/CRC, Boca Raton, Fla, USA, 2008.

[6] M. DiPippo and M. R. S. Kulenović, "Global dynamics of three anticompetitive systems of difference equations in the plane," Discrete Dynamics in Nature and Society, vol. 2013, Article ID 751594, 11 pages, 2013.

[7] M. M. El-Dessoky, "Qualitative behavior of rational difference equation of big order," Discrete Dynamics in Nature and Society, vol. 2013, Article ID 495838, 6 pages, 2013.

[8] H. El-Metwally, "Solutions form for some rational systems of difference equations," Discrete Dynamics in Nature and Society, vol. 2013, Article ID 903593, 10 pages, 2013.

[9] S. Kalabušić, M. R. S. Kulenović, and E. Pilav, "Multiple attractors for a competitive system of rational difference equations in the plane," Abstract and Applied Analysis, vol. 2011, Article ID 295308, 35 pages, 2011.

[10] V. Van Khuong and L. Hong Lan, "Dynamics of certain anti-competitive systems of rational difference equations in the plane," International Journal of Mathematical Sciences and Engineering Applications, vol. 6, no. 5, pp. 417-427, 2012.

[11] M. R. S. Kulenović and G. Ladas, Dynamics of Second Order Rational Difference Equations: With Open Problems and Conjectures, Chapman \& Hall, Boca Raton, Fla, USA, 2002.

[12] K. Liu, Z. Zhao, X. Li, and P. Li, "More on three-dimensional systems of rational difference equations," Discrete Dynamics in Nature and Society, vol. 2011, Article ID 178483, 9 pages, 2011.

[13] S. Moranjkić and Z. Nurkanović, "Basins of attraction of certain rational anti-competitive system of difference equations in the plane," Advances in Difference Equations, vol. 2012, article 153, 19 pages, 2012.

[14] G. Papaschinopoulos, G. Stefanidou, and C. J. Schinas, "Boundedness, attractivity, and stability of a rational difference equation with two periodic coefficients," Discrete Dynamics in Nature and Society, vol. 2009, Article ID 973714, 23 pages, 2009.

[15] Q. Zhang, J. Liu, and Z. Luo, "Dynamical behavior of a system of third-order rational difference equation," Discrete Dynamics in Nature and Society, vol. 2015, Article ID 530453, 6 pages, 2015. 


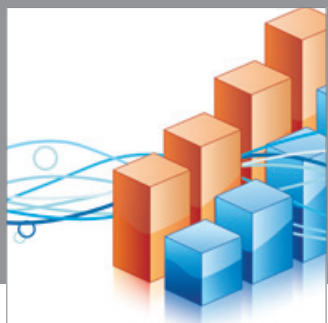

Advances in

Operations Research

mansans

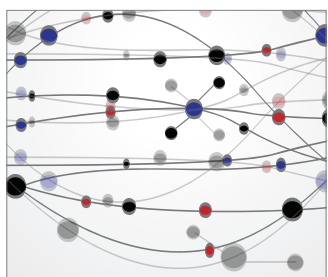

The Scientific World Journal
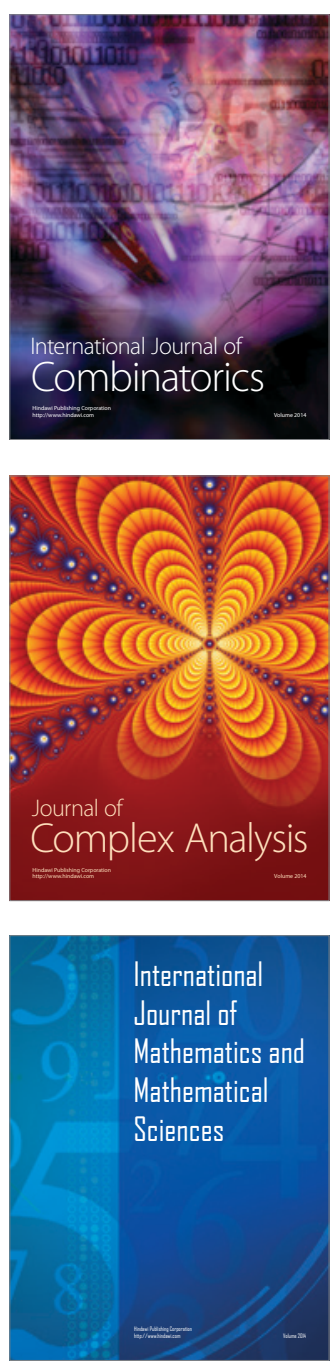
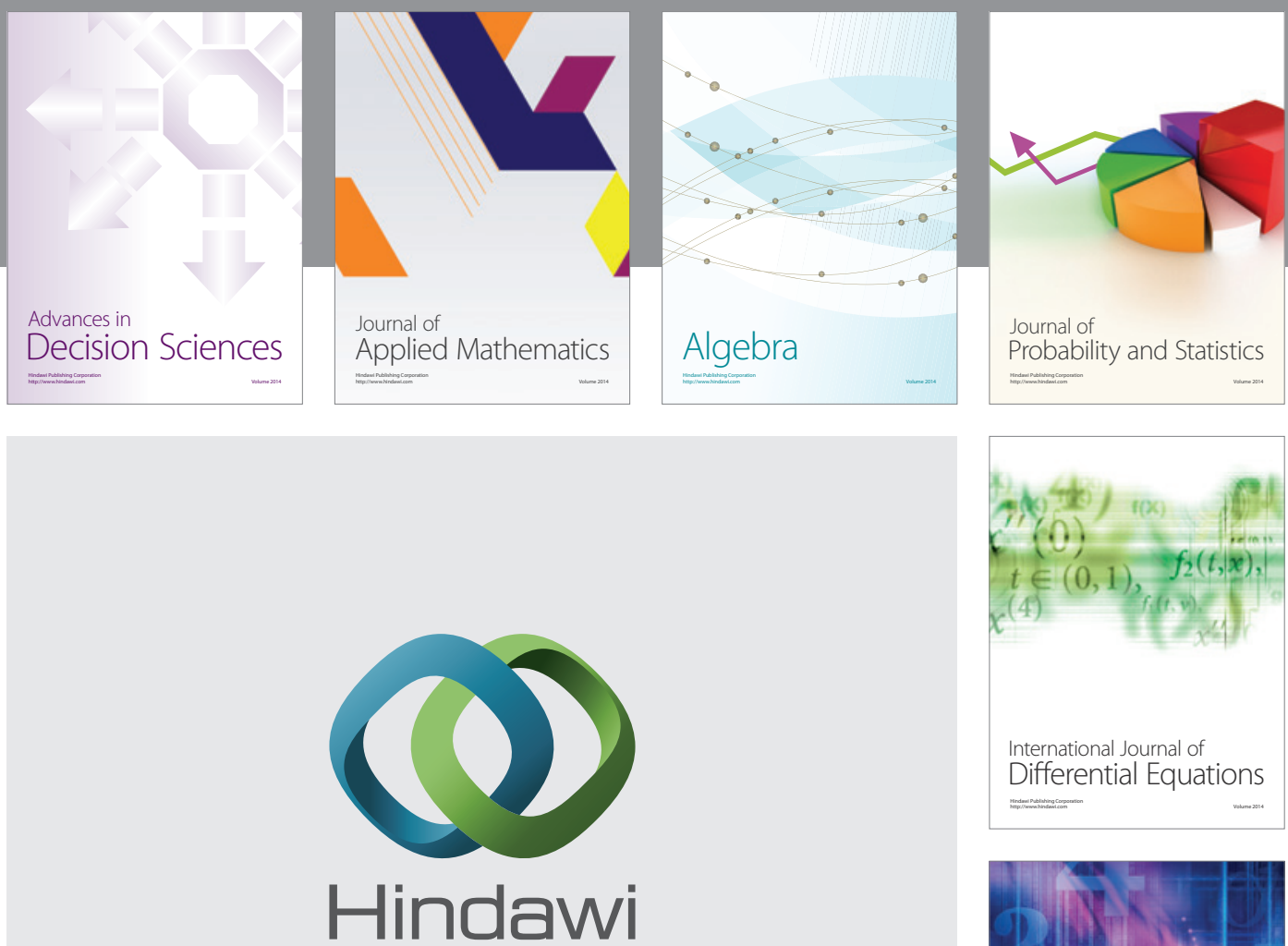

Submit your manuscripts at http://www.hindawi.com
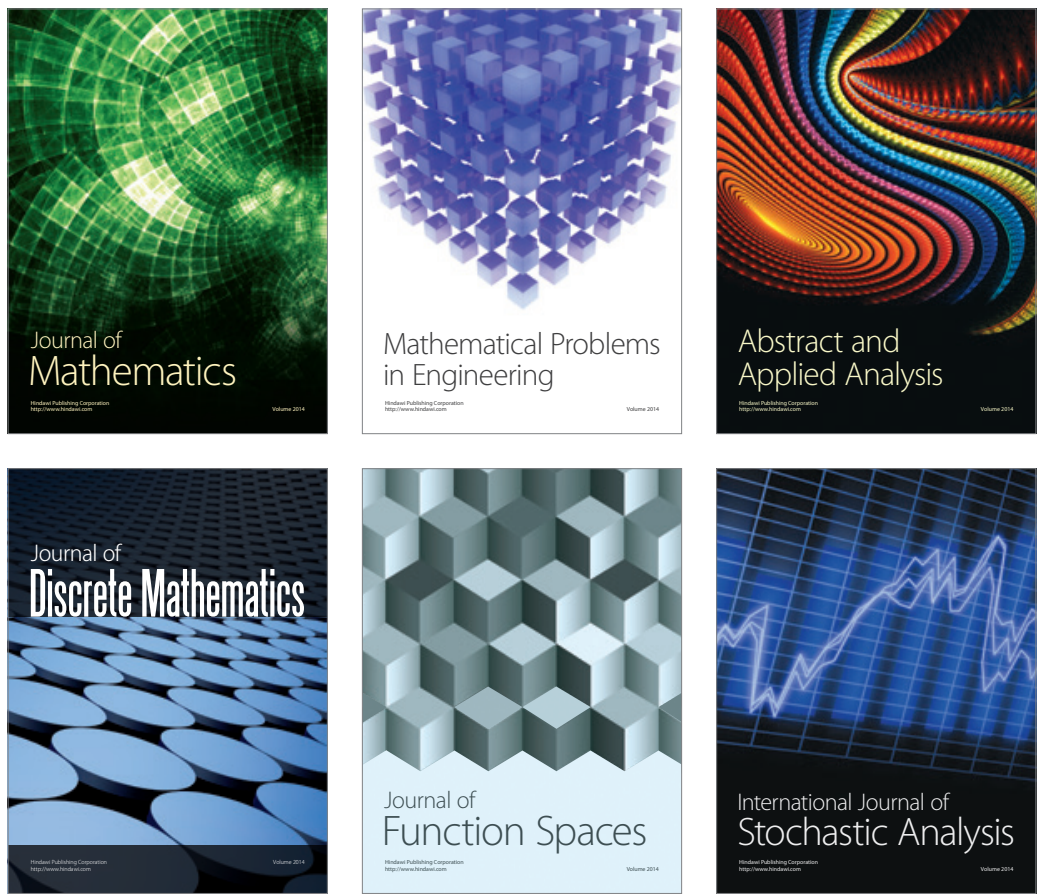

Journal of

Function Spaces

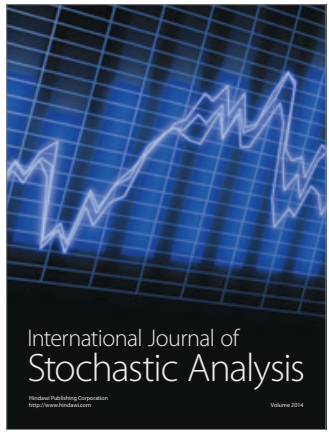

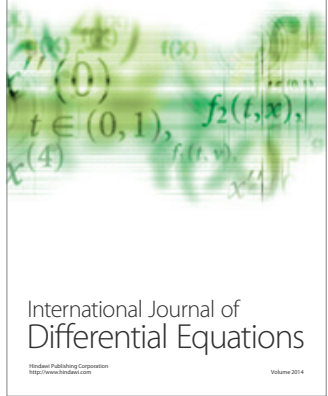
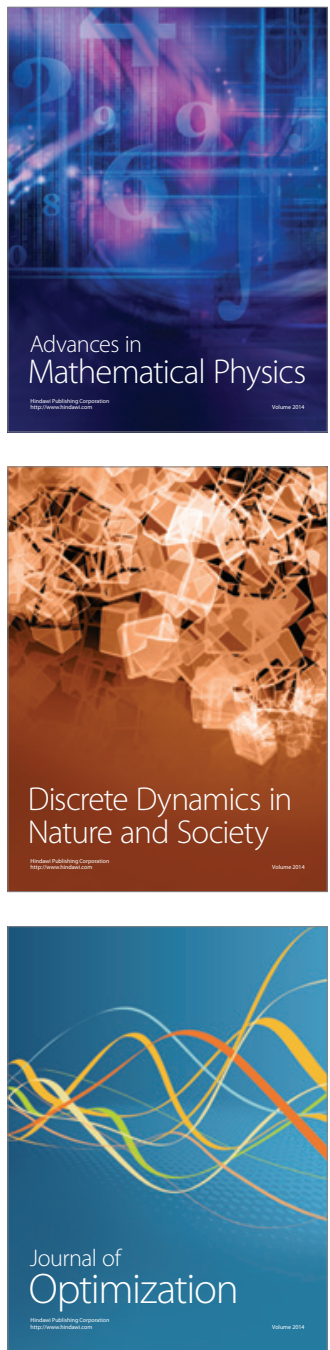